\title{
Monitoring of Electric Buses within an Urban Smart City Environment
}

\author{
José Javier Astrain, Francisco Falcone, Senior Member IEEE, Antonio J. Lopez-Martin, \\ Senior Member IEEE, Pablo Sanchis, Senior Member IEEE, Jesús Villadangos, Ignacio R. \\ Matias, IEEE Senior Member
}

Abstract-A practical experience on monitoring the data generated by electric buses is presented, focusing on energy consumption, charge and state of the batteries. The work is carried out in the framework of a global smart city strategy developed by the H2020 Smart City Lighthouse STARDUST project. The crucial role of the data collection and transmission from electric buses has become evident in this work, so the adopted solutions are covered in detail. A practical electric bus charging station configuration is considered, operating within the city of Pamplona, Spain, with an urban route setting in which electric charging is performed. Various key factors for the practical implementation of the necessary communication infrastructure, including wireless Low Power Wide Area connectivity challenges within the urban scenario settings, based in LoRa/LoRaWAN communication system nodes. The monitoring system architecture is also presented, in which specific machine learning modules in order to collect patterns and visualization of data to enhance planning, operation and maintenance procedures.

Index Terms - business intelligence, electric bus, LoRa, smart city, smart grid

\section{INTRODUCTION}

$\mathrm{S}$ sensors are the foundation of the operation of a smart city gathering data and sending where they can be processed [1]. One possibility could be to utilize cloud computing but it is also posible to delegates its responsibilities to edge computing devices through fog computing, to reduce consumption, greater security, less maintenance, etc. $[2,3]$. In any case, smart cities employ sensors to measure a large number of parameters related to air and water quality, energy usage, traffic flow, etc. These sensors are deployed throughout the city and provide valuable information to policy makers, public administrations or directly to citizens in real time, enabling the city and their citizens to be connected in an urban smart and rational city environment.

The European Commission is committed to supporting

Manuscript received March 1, 2021; accepted XXX. Date of publication XXXX; date of current version XXX. This work was supported in part by the Ministerio de Ciencia, Innovación y Universidades, Gobierno de España (MCIU/AEI/FEDER, UE) under Project Project RTI2018-095499-B-C31, and in part by the European Union's Horizon 2020 Research and Innovation Programme (StardustHolistic and Integrated Urban Model for Smart Cities) under Grant №774094.

An earlier version of this manuscript was presented at the IEEE Sensors 2020 Conference and was published in it's proceedings; https://ieeexplore.ieee.org/document/9278791

J.J. Astrain and J. Villadangos are with the Department of Statistics, Computer Science and Mathematics, Public University of Navarre, 31006 Pamplona, Spain, (e-mail: josej.astrain@unavarra.es; jesusv@unavarra.es).

F. Falcone, A.J. Lopez-Martin, P. Sanchis and I.R. Matias are with the Department of Electrical, Electronic and Communication Engineering, Public University of Navarre, 31006 Pamplona, Spain, (email: francisco.falcone@unavarra.es; antonio.lopez@unavarra.es; pablo.sanchis@unavarra.es; natxo@unavarra.es).

All the authors are also with the Institute of Smart Cities, Public University of Navarre, 31006 Pamplona, Spain. initiatives that represent an advance in the sustainability of its cities. In this framework, this work presents some results of the Smart Cities and Communities Lighthouse STARDUST project [4] in which seven cities are collaborating closely in order to test and validate technical solutions and innovative business models and deliver blueprints for replication throughout Europe and abroad. The objective of the STARDUST project is to pave the way towards low carbon, highly efficient, intelligent and citizen-oriented cities by developing urban solutions and new business models, integrating the domains of buildings, mobility and efficient energy through information and communication technologies (ICT). The aim to be achieved is to test and validate these solutions allowing their rapid implementation in the market. The STARDUST project is showing that the adequate integration of these actions, together with other accompanying non-technological measures, can provide a valid platform to encourage the participation of citizens. The ability to share information from multiple inputs and the cooperation among citizens themselves will increase their quality of life while boosting local economies through a novel productive model based on eco-innovation technologies.

In this context, the transformation of public transport towards more sustainable vehicles is a key aspect to improve, among others, air quality and noise pollution in cities. In this sense, smart and electric buses represent a clear benefit that is identified to a different extent with all the challenges shown in Fig. 1. In addition to contributing to the reduction of atmospheric and acoustic emissions (environmental), electric buses are generally associated with greater comfort and information (social) for users [5] as well as a better use of available energy, just to mention a few of the challenges.

This work aims to contribute to the development of the different aspects that are dealt within the STURDUST project and it has been structured as follows. Section II describes the 
advantages of electric buses and describes the works carried out on one of the bus lines in one of the cities participating in the project. As can be deduced from section II, the communication and monitoring of the data that is collected are fundamental tasks to optimize the charging infrastructure and, ultimately, integrate the information obtained into the city's platform for the benefit of the citizen. In this sense, sections III and IV describe the solutions adopted for communication and data monitoring, respectively. Finally, conclusions are presented in Section V.

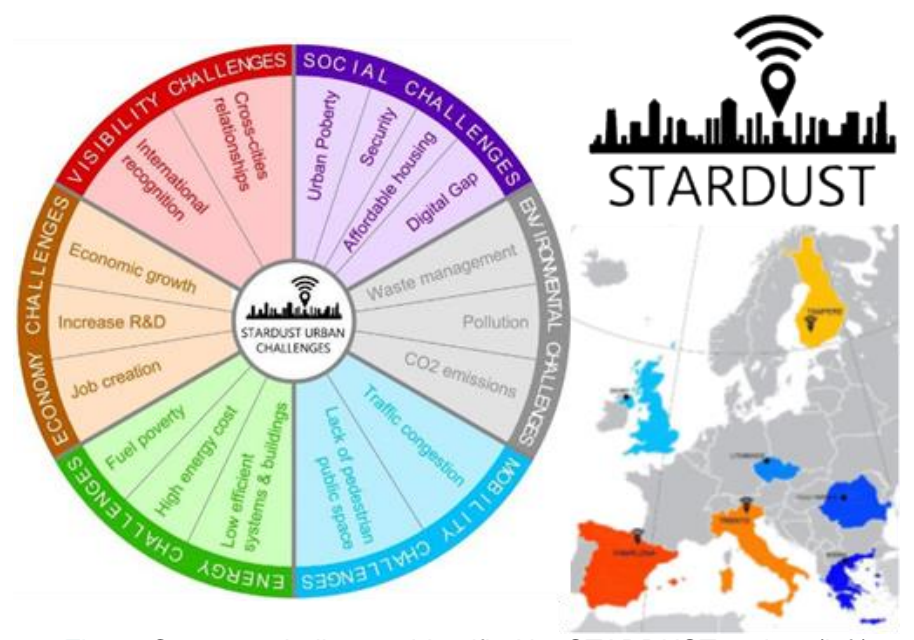

Fig. 1. Common challenges identified by STARDUST project (left) and the associated cities (right).

\section{Electric Buses AND SMART GRIDS}

In a context in which countries such as Norway, The Netherlands, Germany, United Kingdom and France are developing plans to ban internal combustion energy vehicles in the next few years, electrical mobility is experiencing a fast development due not only to the technical improvements on electric vehicles but also to the price reduction and energy density increase of the batteries [6]. This transport electrification is a key element to achieve smart and sustainable cities. Electric vehicles offer improved air quality, noise pollution reduction, lower energy consumption and, if electric energy is obtained from renewable sources, energy independence. Due to their higher operation hours and passengers per vehicle, electric buses can have the greatest impact on the achievement of these advantages in the future smart cities [7].

However, the operation of electric buses entails significant challenges for both the electricity distribution network in cities and for the energy management of bus batteries. In order to reduce the weight of the onboard storage system, electric buses optimize the size of their batteries so that they are charged multiple times throughout the day, usually at the end of each trip. This operation significantly reduces the size of the storage but has two important consequences. Firstly, it involves high battery cycling, which can reduce battery life. To minimize this problem, the size of the batteries is designed by anticipating a maximum discharge per trip of around 20$30 \%$. Secondly, the bus must recharge its batteries in a very short time taking advantage of the stop time at the head stations of each route, which means that the electricity demanded from the electricity distribution grid experience a significant power peak that can significantly disturb grid voltage stability affecting especially the consumers near the connection point. In addition, this problem is exacerbated as electric urban transport lines and charging stations increase.

The characteristics of public bus transportation, and especially their predictable routes and schedules, make it possible to develop specific infrastructure concerning both the vehicle itself and the charging points. Firstly, optimized energy management algorithms can be designed for the bus batteries in order to improve their life expectancy and performance. Secondly, the required charging infrastructure can be properly distributed along the city depending on the expected electric demand and the characteristics of the local electric distribution grid, and operated under dedicated management algorithms [8]. This charging infrastructure should include stationary battery-based support charge stations that can be built close to the charging points of the buses and designed so as to manage the power peaks demanded by the buses and reduce their impact on the distribution grid. All this charging infrastructure configures a global Smart Grid that is fundamental to progressively integrate the new electric transportation paradigm including not only buses but also taxis, shared public electric bicycles and other means of transport.

Different approaches have been presented in relation with bus charging infrastructure implementation and management, such as the simultaneous planning of electric transit route network and fast charging stations with the aid of modified genetic algorithms [9], the use of deep learning algorithms to optimize wireless power transfer systems coupled to electric bus fuel cell charging [10], battery size reduction based on specific cost optimization algorithms [11] or the study of resilient electric bus operation in the case of disaster events [12], among others. For an optimal operation of both the electrical storage system of the buses and the operation of this Smart Grid, advanced monitorization and communication systems are required.

The city of Pamplona, located in the north of Spain, has been fostering sustainable public transport such as integrated biogas and hybrid buses for years. Since March 2019, Route 9 is fully operated by electric buses in a pioneering initiative in Spain. Particularly, six electric buses cover the $12.3 \mathrm{~km}$ distance of the go and return trip, departing every 12 minutes from the two terminals, located one at the Arrosadia Campus of the Public University of Navarra (UPNA) and the other one at the Train Station. The buses have a $44.3 \mathrm{kWh}$ Lithium Titanate (LTO) battery, with a rated voltage of $650 \mathrm{~V}$ and a weight of $550 \mathrm{~kg}$. Both terminals have a fast charging station, typically used in public transportation where the buses have to be quickly charged during the time stops. The energy consumption per trip is around $10 \mathrm{kWh}$ and the bus has about 3.5 minutes to charge again its battery. This energy consumption represents around $20-25 \%$ of the rated capacity of the batteries. Fig. 2 shows the charging station at the Public University of Navarra (UPNA) Campus, when the pantograph is connected to the bus. 


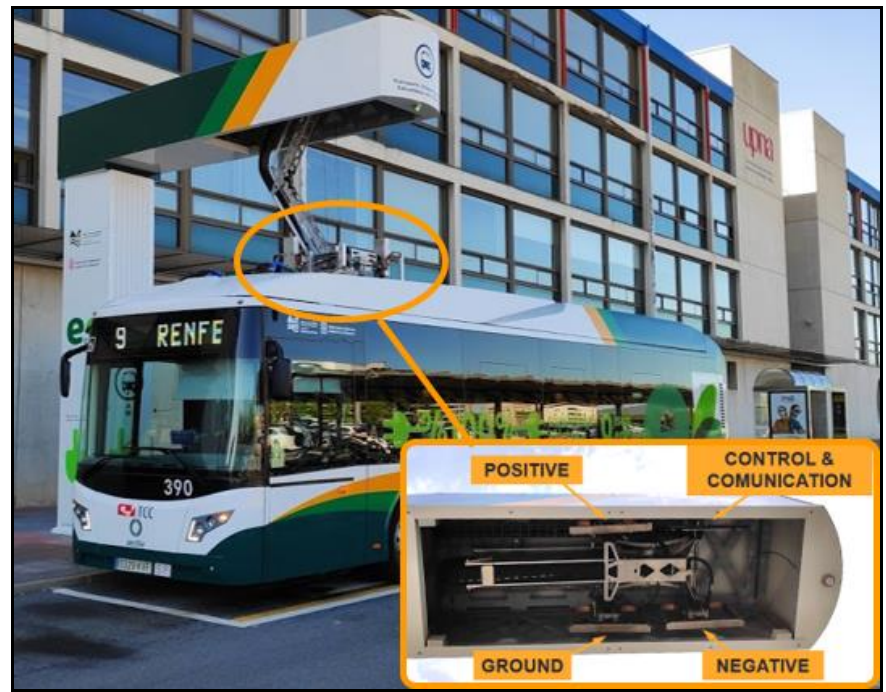

Fig. 2. Bus charging station at Public University of Navarra Campus.

In spite of the positive impact that the electric buses have on the city, their electric supply is not straightforward. As mentioned before, the electric peaks demanded by the charging stations can compromise the quality of the electric distribution network causing voltage drops and perturbations. The charging peaks of the electric buses analyzed in this paper can reach values of around $250 \mathrm{~kW}$, while the average consumed power can be lower than $70 \mathrm{~kW}$. Fig. 3 shows an example of the charge electric power on a typical day. The gaps in the profile are due to the fact that some charges are missed when the stop time is not long enough. In addition, charging time can vary depending on the battery state of charge (SOC) at this moment. The charge electric power demanded by the buses is measured once per second by means of a Precision Power Analyzer WT1800 from Yokogawa which is connected at the $\mathrm{AC}$ input terminals of the bus charging station. Measured data have been continuously collected since March 2020.

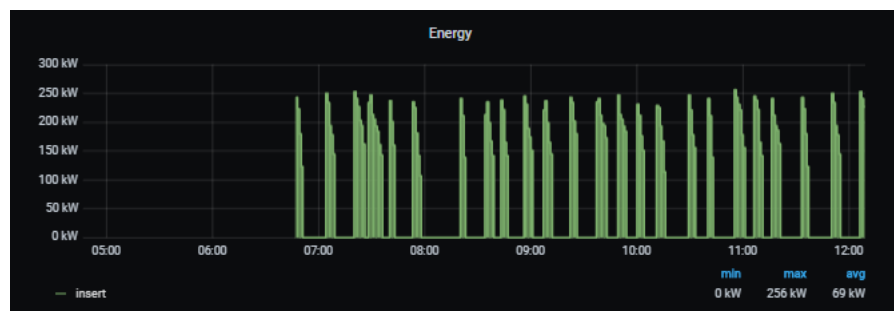

Fig. 3. Typical charge electric power graph of the electric buses at UPNA. Charging peaks can reach values around 250 $\mathrm{kW}$.

In order to avoid demanding electric peaks from the distribution network, a stationary support battery is planned to be included at the UPNA bus charging station [13]. This battery would be responsible for providing the peak power to the buses during the charging time and would charge at a constant power from the distribution network for the rest of the time. In order to develop optimized energy management strategies for this stationary support battery, the communication system of the global electric bus network has to provide in real time the needs of the next bus to be charged in terms of required energy, expected stop time and bus battery performance. This aspect is analyzed in the next section.

\section{COMMUnication SYSTEM}

In order to enable data exchange between electric buses and infrastructure nodes, wireless communications based on lowpower wide-area network (LPWAN) standards have been employed. In terms of system level requirements, low transmission rates (below $50 \mathrm{Kbps}$ ) and moderate latency values (in the range of 2-5 seconds) are compliant quality of service metrics. Moreover, long distance communication range is compulsory in order to optimize node number, configuration and location. It is worth noting that the proposed communication scheme corresponds to vehicle to infrastructure links, in which mobile nodes located within the urban electric buses communicate with the infrastructure nodes, distributed within the city layout. With these settings, LoRa/LoRaWAN communications have been considered, owing to their long range, low power consumption and ease to integrate gateway nodes into cloud enabled infrastructures.

The supported network topology is a star topology, in which the nodes located within the urban buses are directly connected to the corresponding gateways, located within urban infrastructure, such as street lamp or municipal buildings. This provides wireless communication links with a relative height difference, minimizing non-line-of-sight conditions usually given in vehicle-to-vehicle links. In this way, functionalities such as mesh networking can be circumvented, reducing complexity and energy consumption at MAC level [14].

To validate the feasibility in the use of LoRa/LoRaWAN for this application, radio channel characterization has been performed, for an operational frequency of $868 \mathrm{MHz}$. The analysis has been performed considering different types of on board nodes: LoRa Dragino, LoRa 3 Click, MWR WAN 1310 and I-NUCLEO-LRWAN1, whereas TTN Gateways are used as infrastructure nodes. Empirical/statistical propagation models within urban scenarios have been employed [15], based on COST 231 model, considering a height setting of 10 $\mathrm{m}$ for the infrastructure node and $2.5 \mathrm{~m}$ for the mobile node. The proposed methodology based on coverage/capacity estimations is schematically depicted in Fig. 4. 


\section{LPWAN System Design}

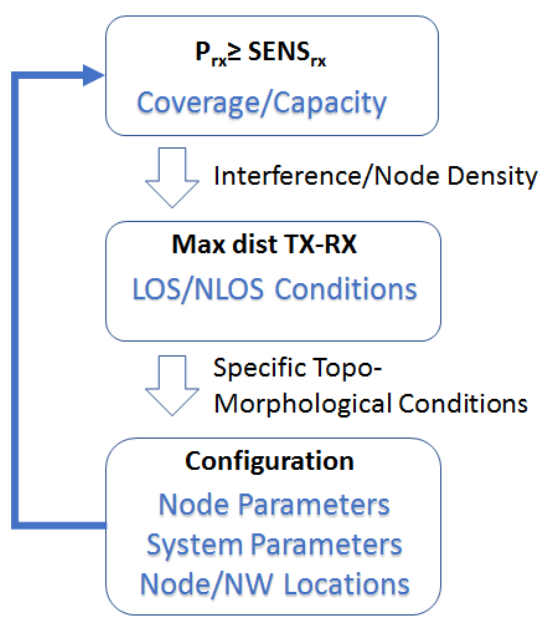

Fig. 4. Schematic overview of coverage/capacity LPWAN system design.

Different wireless link conditions, given geometrical disposition of infrastructure node and mobile node in terms of grazing angle have been considered, spanning from $35^{\circ}$ (Case 1), $55^{\circ}$ (Case 2) and $75^{\circ}$ (Case 3). Coverage/capacity relations for these cases with LoRa@868MHz is depicted in Fig. 5.

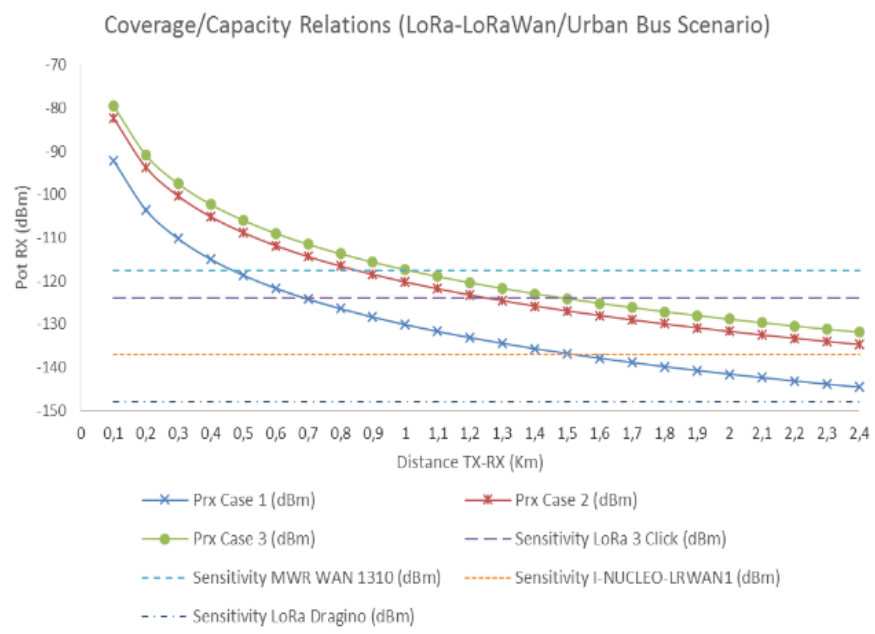

Fig. 5. Coverage/capacity estimations for infrastructure to vehicle links employing LoRa/LoRaWAN at $868 \mathrm{MHz}$, for different transceivers and link conditions.

As it can be seen, worst case conditions provide viable communication links in the range of $400 \mathrm{~m}$, whereas less severe conditions and the use of lower sensitivity transceivers provide communication links in excess of $2 \mathrm{Km}$. With this in mind and considering that the operational service area within the city of Pamplona spans in $25.1 \mathrm{~km}^{2}$, service can be provided with overlapping coverage with 3 infrastructure gateways. Quality of service metrics can also be analyzed by obtaining bit error rate (BER) values as a function of infrastructure to vehicle distance, which is depicted in Fig. 6, for the three different geometrical link configurations. Note that larger grazing angles lead to improved BER at low distances, as expected from Fig. 5.

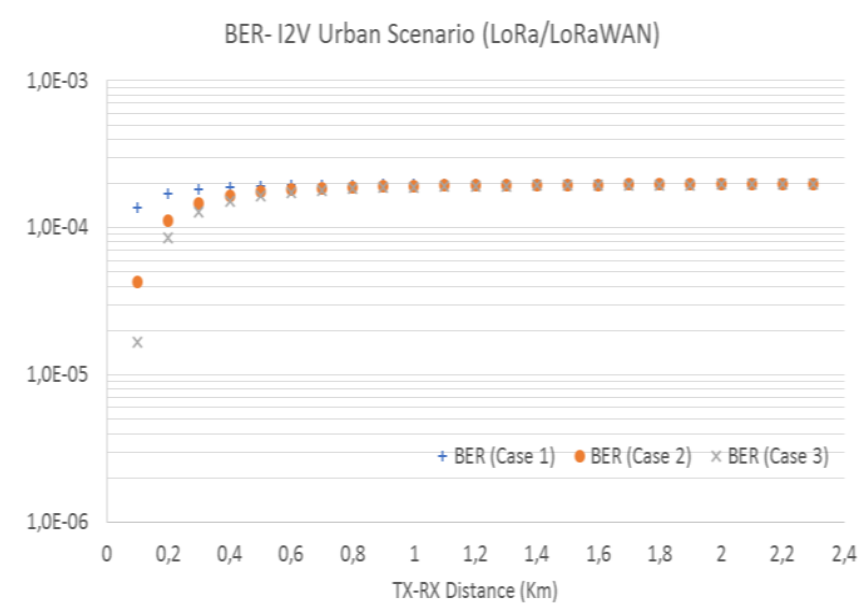

Fig. 6. BER estimation for infrastructure to vehicle links employing LoRa/LoRaWAN at $868 \mathrm{MHz}$, for different transceivers and link conditions.

The operation of the LoRa/LoRaWAN links will be affected by other elements embedded within the urban clutter, such as diffuse scattering given by surface roughness from building facades or the impact of vegetation present within the communication links. The impact of the later is shown for the case of the I2V links to the buses, modelled with the help of the Weissberger model [16], and demonstrated by the following expression:

$$
L_{W}=1.35 f^{0,284} d^{0,558}, \quad 14 m<d<400 m
$$

where $d$ is the distance in meters between transceivers and $f$ is the operating frequency in $\mathrm{GHz}$.

The results, depicted in Fig. 7 exhibiting additional losses, as a function of link distance, which can be in excess of $30 \mathrm{~dB}$ for link distances in the $400 \mathrm{~m}$ range, which strongly impacts the operation of the system. Gateway deployment in these conditions leads to coverage areas in the range of $0.4 \mathrm{~km}^{2}$, which implies shifting from initial 3 nodes for city coverage to up to 62 gateway nodes to comply with coverage/capacity conditions. The increase in path loss directly affects coverage/capacity relations, with higher BER values in the case of presence of vegetation within the link path, as shown in Fig. 8. An equivalent analysis can also be performed considering effects derived from diffuse scattering, which is given by surface roughness conditions, a case that occurs in the presence of non-homogeneous building facades (e.g., presence of bricks or other structural elements). As an example, considering diffuse scattering modeled by Kirchoff model [17], the impact on received signal levels in the I2V communication scenario considered in this work can vary up to $50 \%$ in reflected components with grazing angles in E-field incidence range between $70^{\circ}-90^{\circ}$. 


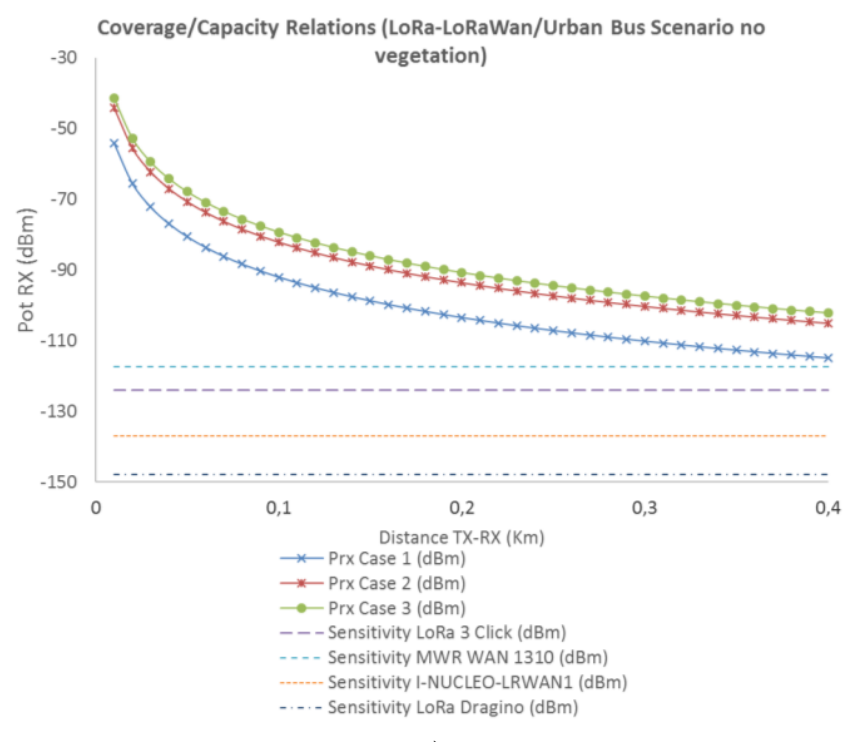

a)

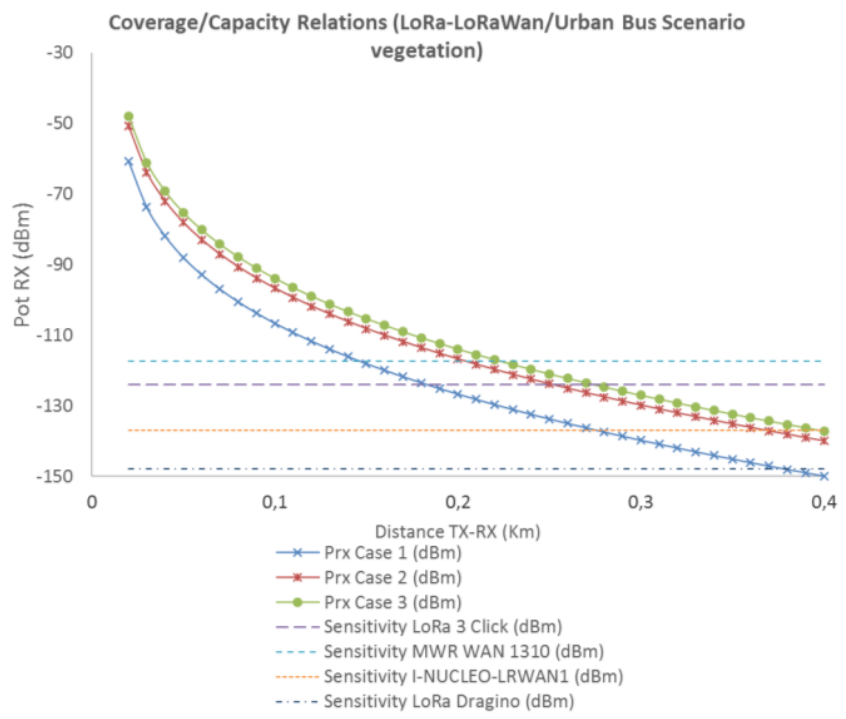

b)

Fig. 7. Coverage/capacity estimations for infrastructure to vehicle links employing LoRa/LoRaWAN at $868 \mathrm{MHz}$, considering: a) low distance/no vegetation conditions, b) dry vegetation within the TX-RX propagation link.

BER- I2V Urban Scenario (LoRa/LoRaWAN)-no vegetation

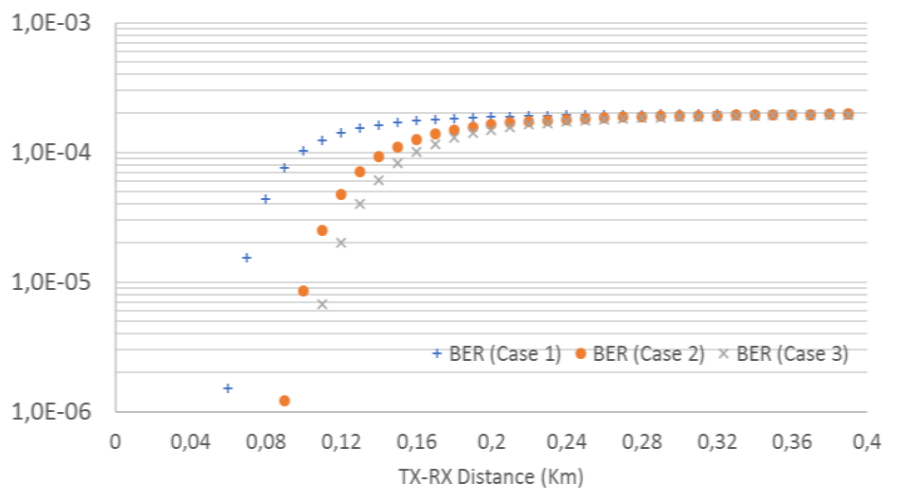

a)

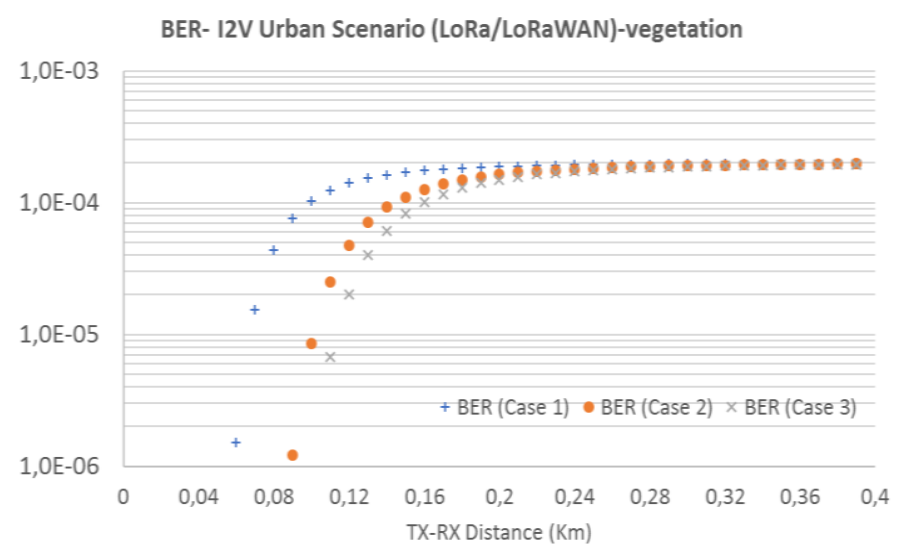

b)

Fig. 8. BER estimation for infrastructure to vehicle links employing LoRa/LoRaWAN at $868 \mathrm{MHz}$, a) low distance/no vegetation conditions, b) dry vegetation within the TX-RX propagation link.

Another relevant aspect to consider is given by potential capabilities of node densification and hence, system scalability supported by LoRa/LoRaWAN devices. System growth is mainly constrained by interference conditions (given by physical node distribution and probability of simultaneous transmissions), as well as by overall simultaneous traffic demands (given by user traffic models and gateway capacity) [18-20].

As previously stated, the initial prototype and pilot based relies on the use of LoRa/LoRaWAN IDE development platform. The subsequent hardware development of the sensor nodes can be carried out in different ways. A popular approach is using platforms, like Arduino and Raspberry Pi, which can expedite design and lead to a fast prototyping phase. In fact, several off-the-shelf hardware boards can be found in the market, including a wide variety of microcontrollers and single-board computers. Wireless connectivity to a low-power wide area network (LPWAN) using LoRa/LoRaWAN can be simply achieved in many of these solutions using dedicated boards (e.g. LoRa Dragino shield or MKR WAN 1310 for Arduino). This approach sacrifices design customization by development time. However, there are so many different types of off-the-shelf devices to choose from that a wide range of configurations are available. Obviously, based on performance optimization only, a fabricated custom board developed specifically to the application at hand is the best option. The development time and cost are higher, but as the monitoring platform is scaled out with several devices, these custom hardware boards become the best alternative considering a cost-performance tradeoff. Fig. 9 shows a simplified diagram of a LoRa/ LoRaWAN sensor node. Signals captured by the sensors are pre-processed in the analog domain (e.g. amplification, filtering, offset and temperature compensation) if required and then they are acquired by the microcontroller unit (MCU). Also, off-the-shelf sensor modules can be directly connected to the $\mathrm{MCU}$ via $\mathrm{I} 2 \mathrm{C}$ interface. The $\mathrm{MCU}$ processes and stores them as needed, forms a LoRaWAN frame and drives the LoRa transceiver to transmit the LoRaWAN data frame. The transceiver is typically in charge 
of the LoRa physical layer protocol, while the MCU manages the LoRaWAN protocol stack, the sensors and the application software. The $868 \mathrm{MHz}$ ISM band is used. Concerning power supply, a rechargeable battery is employed as energy storage element. Optionally, energy harvesting can be employed to recharge the battery, increasing the battery lifetime. The most suitable energy harvesting transducers on the bus are photovoltaic cells (when the sensor node is placed on the roof top) and piezoelectric, electrostatic or electromagnetic transducers to capture energy from vibrations caused by the movement of the bus. The initial implementation considers the inclusion of power meter sensors for battery charging/management operations. The scalability of the sensor node platform enables the inclusion of additional analog/digital sensors as a function of future requirements.

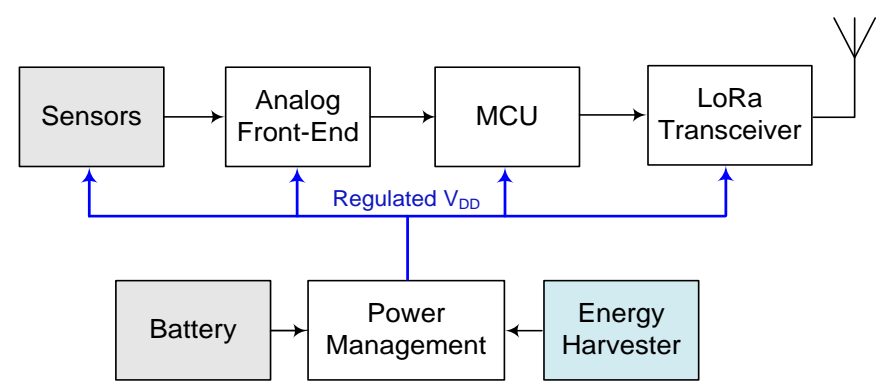

Fig. 9. Schematic diagram of the wireless sensing node.

\section{MONITORING APPROACH}

Cloud-based monitoring systems, which usually include both vehicle tracking and operation monitoring, are designed specifically to improve the daily operations of city buses. Buses include a communication gateway, which sends, periodically or on-demand, operation and location information to the management system. Data gathering is performed through the LoRa connectivity of the measurement devices located on the bus and on the pantographs located at the start and end of the transportation line. Location data is used to grant bus tracking for the purpose of notifying users when the vehicle is expected to arrive at each bus stop, but also to study the overall traffic flows in order to optimize the city's traffic and ensure urban transport efficiency. Data collection is performed Data operation includes the estimated occupation of the vehicle, the flow of passengers getting on or off the bus at each bus stop, the temperature and humidity of the vehicle, the incidents that the driver wishes to record, the notification of traffic incidents or of the vehicle itself, and many others. In our case, we include additional information regarding the monitoring of the vehicle's batteries. The aim is to monitor the vehicle's autonomy, in order to achieve an efficient consumption and optimize charge cycles of the batteries, but also to carry out the least possible number of recharges and thus achieve a longer life of the batteries.

The monitoring system deployed is a cloud-based solution over a set of VMware virtualized Ubuntu 18 machines. The monitorization system, depicted in Fig. 10, has four layers. The data acquisition layer includes two Apache components called NiFi and Kafka, which are in charge of large quantities of data acquisition from buses (via LoRa, as described in the previous section). $\mathrm{NiFi}[21]$ transforms data to allow its proper storage into a MySQL [22] database. Apache Kafka [23] follows the subscription-publication paradigm on messaging queues and allows offering the acquired (published) data at the disposal of the subscribed MySQL database. Data is stored in our case into a MySQL relational database, and finally, the Visualization Layer includes a Grafana [24] analytics visualization web application, a map visualization tool based on the Leaflet Javascript library [25] and Swagger [26] to provide a RESTful web service for data publishing. The Machine Learning Layer includes the use of TensoFlow [27] and Keras [28] open source libraries, which allow analytics and machine learning processing pipelines. Data collected is analyzed using Machine Learning (ML) models previously generated in batch in conjunction with historic and persistent data that can be accessed in real time from APIs in Java, R and Python, to implement customized algorithms, statistics, time series analysis or classification and clustering algorithms

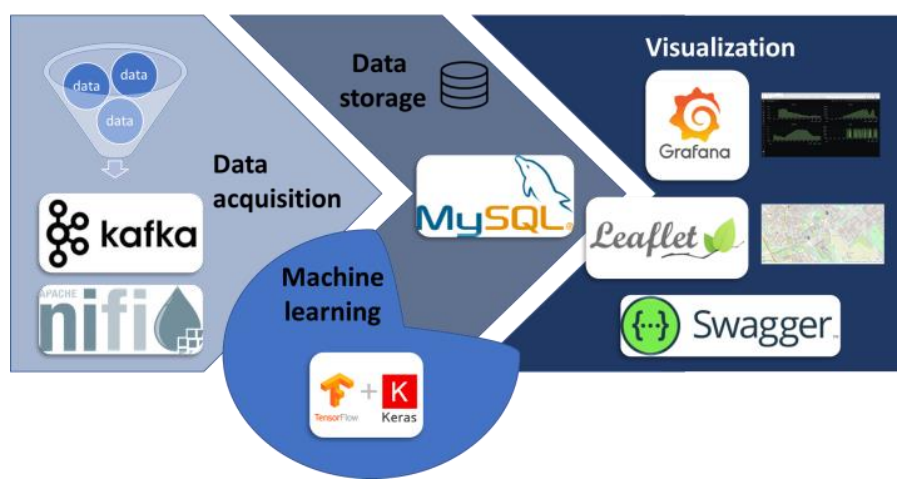

Fig. 10 Monitorization system architecture

As an example, Fig. 11 illustrates one of the monitoring dashboards developed. It the average and maximum power of each charge, the elapsed time between charges, the energy consumption, temperature, relative humidity, insolation and energy charge information. In particular, the obtained charging profiles can be exploited as described in Section II.
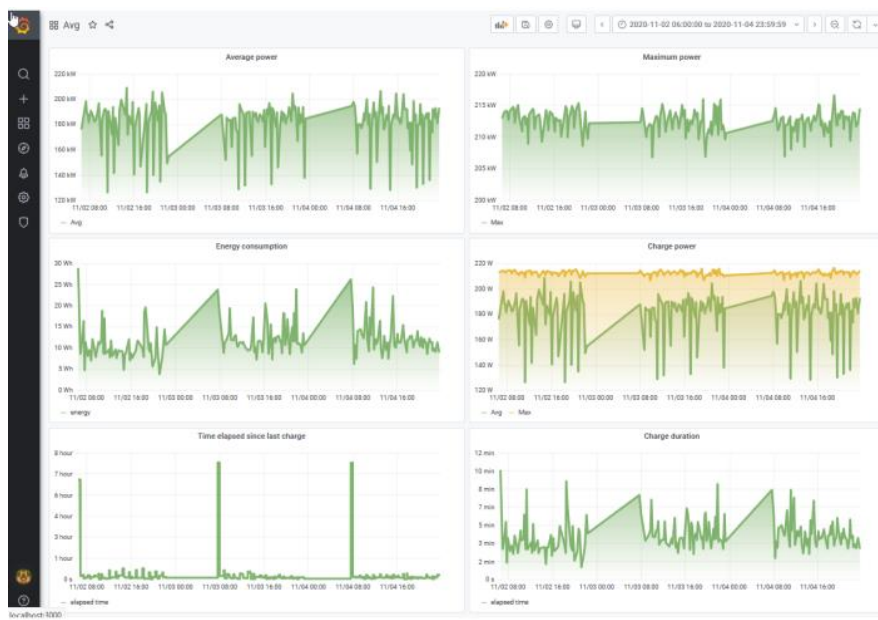

Fig. 11 Monitorization dashboard (Grafana).

Fig. 12 shows in more detail the information provided by the proposed monitoring system. This information allows for a better understanding of both the recharging and operating 
cycles of the buses. This allows the entire life cycle of the buses and of the recharging systems to be monitored. The system allows dynamic selection of the monitoring periods, which facilitates the search for and identification of behavioral patterns and anomalies, and the automated creation of reports.
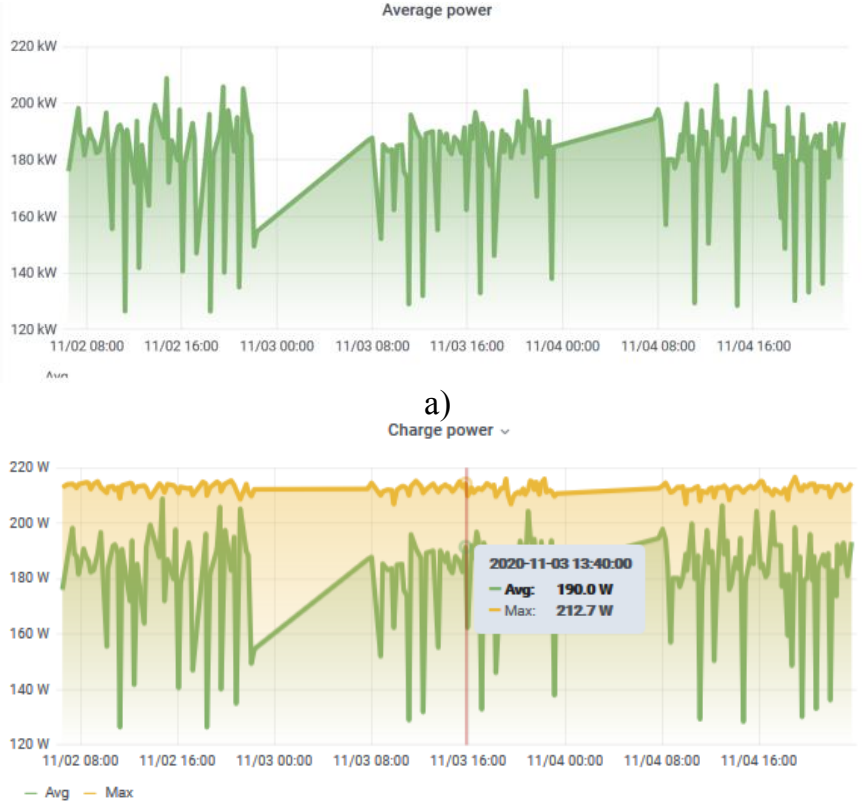

b)

Fig. 12 Dashboard graphics: a) average power of a charge and b) use of the cursors to obtain specific values.

The system includes a machine learning module, which aims to support technicians in the detection of operating patterns, as well as to prevent system failures due to out-ofrange operations, facilitate predictive maintenance and, in short, help in the proper decision-making in the operation of the electric bus fleet and the recharging system. Keras provides an API to create and train deep learning models with TensorFlow. Both together allow the analysis of monitoring data sets to identify such patterns of operation. The ML module uses a set of convolutional neural networks (CNNs), which perform well when analyzing visual imagery. In our case, the monitoring system provides, among others, the graphs of power consumption and electric charge. These images feed into a set of CNNs, which are trained to identify different patterns and address different issues. Although each network may have some differences from the others, these CNNs basically consist of an input layer, whose size is determined by the size of the graphs (the higher the resolution of the graphs the higher the number of input neurons), followed by two Conv2D-Max_Pooling2D layers, followed by a flatten layer, and then followed by two Dense (ReLU and SoftMax)-Dropout layers. As a result, the obtained networks can analyze the values provided in real time by the monitoring system and show whether or not a malfunction is occurring, and identify operation patterns.

We capture the graphs of the monitoring system we are interested in on an hourly and daily basis with the aid of a script and we use these graphs to build a set of training samples. These samples are labeled with the help of experts and used to train each network with its corresponding training set (electrical recharges, power consumption, path lengths...).

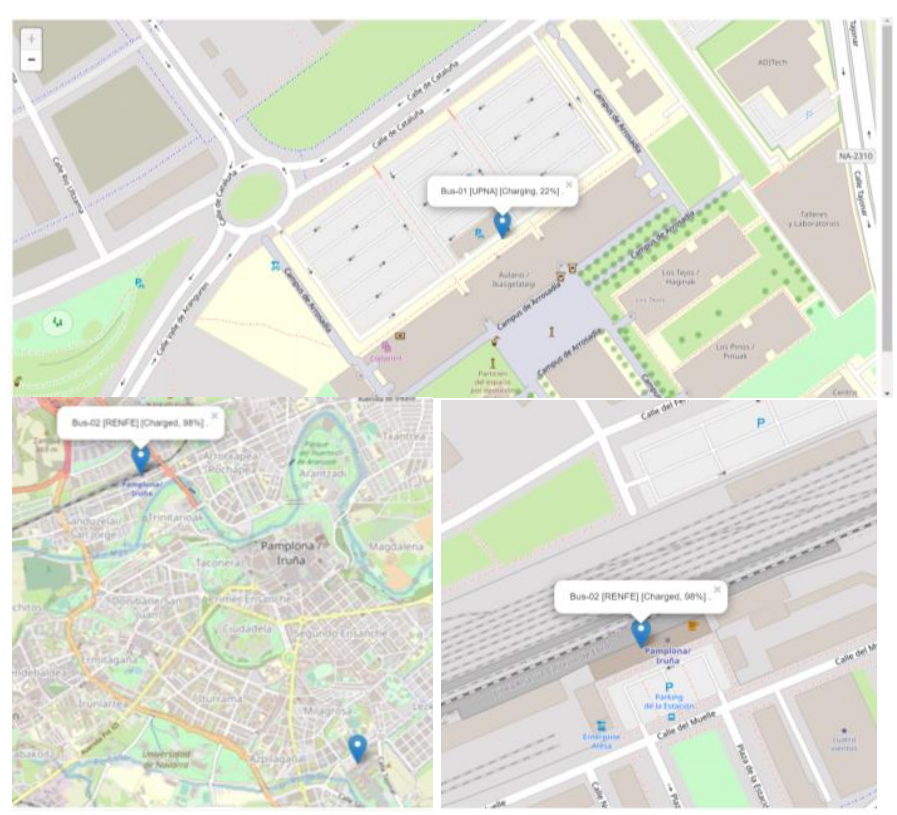

Fig. 13 Monitorization dashboard (Grafana).

The dashboard is complemented by an interactive map that shows in real time the location of the vehicles, whether or not they are charging their batteries, and their charge level. This interface is very easy to implement with the help of the Leaflet library [25]. Fig. 13 illustrates the graphical interface. In this case, the first bus is charging at the bus stop located at UPNA and its battery is at $22 \%$ of capacity, while the second one is charged (98\%) at RENFE's bus stop. The blue indicators show that both buses are stationary, they are about to start their journeys and they are on schedule.

\section{CONCLUSION}

Within the European Stardust project, this work shows some results related to the integration to the city platform of the sensors data generated in electric buses, including the monitoring of the state of the batteries. These data are relevant to increase the life of the batteries and to be able to optimize the operation of the charging stations and the charging cycles of the buses in the city. In order to develop energy management strategies that are truly optimized, it is necessary to have a communication system that provides, in real time, the needs of the next bus to be charged in terms of energy required, expected stop time, status of the batery, etc., including machine learning modules that collect patterns from the system itself and finetune needs that can reduce decision-making times. Finally, a complete communication coverage analysis considering different aspects has been also presented We hope that this experience will be useful to other cities and contribute to the development and integration of electric buses into smart cities in a practical manner.

\section{ACKNOWLEDGMENT}

The authors want to thank the European Commission for financial support through the STARDUST project, and specifically the local partners involved such as the Pamplona City Council, Mancomunidad de la Comarca de Pamplona 
(MCP), National Renewable Energy Centre of Spain (CENER) and Sociedad Ibérica de Construcciones Eléctricas, S.A. (SICE).

\section{REFERENCES}

[1] R. Morello, S. C. Mukhopadhyay, Z. Liu, D. Slomovitz and S. R. Samantaray, "Advances on Sensing Technologies for Smart Cities and Power Grids: A Review," in IEEE Sensors Journal, vol. 17, no. 23, pp. 7596-7610, 1 Dec.1, 2017, doi: 10.1109/JSEN.2017.2735539.

[2] T. Kanaya, A. Nakao, S. Yamamoto, M. Oguchi and S. Yamaguchi, "Edge Computing for IoT Sensors Based on DPN," 2020 IEEE International Conference on Consumer Electronics - Taiwan (ICCETaiwan), Taoyuan, Taiwan, 2020, pp. 1-2, doi: 10.1109/ICCETaiwan49838.2020.9258322.

[3] Q. Qi and F. Tao, "A Smart Manufacturing Service System Based on Edge Computing, Fog Computing, and Cloud Computing," in IEEE Access, vol. 7, pp. 86769-86777, 2019, doi: 10.1109/ACCESS.2019.2923610.

[4] STARDUST project, https://stardustproject.eu/ (accessed Feb 27, 2021).

[5] A. Ghosh, P. Pramanik, K. D. Banerjee, A. Roy, S. Nandi and S. Saha, "Analyzing Correlation Between Air and Noise Pollution with Influence on Air Quality Prediction," 2018 IEEE International Conference on Data Mining Workshops (ICDMW), Singapore, Singapore, 2018, pp. 913918, doi: 10.1109/ICDMW.2018.00133.

[6] BloombergNEF, "Electric Vehicle Outlook 2020", https://about.bnef.com/electric-vehicle-outlook/ (accessed June 27, 2020).

[7] A. Nightingale, "Forget Tesla, It's China's E-Buses That Are Denting Oil Demand", Bloomberg, 2019. https://www.bloomberg.com/news/articles/2019-03-19/forget-tesla-it-schina-s-e-buses-that-are-denting-oil-demand (accessed June 27, 2020).

[8] A. M. Othman, H. A. Gabbar, F. Pino, M. Repetto, "Optimal electrical fast charging stations by enhanced descent gradient and Voronoi diagram”, Comput. Electr. Eng., vol. 83, p. 106574, 2020.

[9] W. Zhang, H. Zhao and Z. Song, "Integrating Transit Route Network Design and Fast Charging Station Planning for Battery Electric Buses," in IEEE Access, vol. 9, pp. 51604-51617, 2021, doi: 10.1109/ACCESS.2021.3068771.

[10] M. Rafiei, J. Boudjadar, M. P. Griffiths and M. -H. Khooban, "Deep Learning-Based Energy Management of an All-Electric City Bus With Wireless Power Transfer," in IEEE Access, vol. 9, pp. 43981-43990, 2021, doi: 10.1109/ACCESS.2021.3066300

[11] M. A. Gormez, E. Haque and Y. Sozer, "Cost Optimization of an Opportunity Charging Bus Network," in IEEE Transactions on Industry Applications, doi: 10.1109/TIA.2021.3061031

[12] B. Li, Y. Chen, W. Wei, S. Huang and S. Mei, "Resilient Restoration of Distribution Systems in Coordination with Electric Bus Scheduling," in IEEE Transactions on Smart Grid, doi: 10.1109/TSG.2021.3060801.

[13] I. Ojer, A. Berrueta, J. Pascual, P. Sanchis, A. Ursúa, "Development of energy management strategies for the sizing of a fast charging station for electric buses", Proc. of $20^{\text {th }}$ IEEE Int. Conf. on Environment and Electrical Engineering, Jun 2020.

[14] D. Zorbas, K. Abdelfadeel, P. Kotzanikolaou, D. Pesch, "TS-LoRa: Time-slotted LoRaWAN for the Industrial Internet of Things", Computer Communications 153 (2020) 1-10

[15] X. Yin, X. Cheng. "Propagation Channel Characterization, Parameter Estimation and Modelling for Wireless Communications.” Ed. John

[16] Wiley \& Sons, Singapore, 2016.

[17] M. A Weissberger, "An initial critical summary of models for predicting the attenuation of radio waves by trees", Dept. Defense, Electromagn. Compatibility Anal. Center, Annapolis, MD, USA, Tech. Rep. EST-TR81-101, Jul. 1982.

[18] A. Molisch, "Wireless Communications", John Wiley \& Sons, 2010.

[19] Haxhibeqiri, F. Van den Abeele, I. Moerman, J. Hoebeke, "LoRa Scalability: A Simulation Model Based on Interference Measurements" Sensors, vol. 17, no. 6, p. 1193, May 2017.
[20] P. J. Basford, F. M. J. Bulot, M. Apetroaie-Cristea, S. J. Cox, and S. J. Ossont, "LoRaWAN for Smart City IoT Deployments: A Long Term Evaluation," Sensors, vol. 20, no. 3, p. 648, Jan. 2020.

[21] Apache Nifi, https://nifi.apache.org/ (accessed February 27, 2021).

[22] MySQL, https://www.mysql.com/ (accessed February 27, 2021).

[23] Apache Kafka, https://kafka.apache.org/ (accessed February 27, 2021).

[24] Grafana, https://grafana.com/ (accessed February 27, 2021).

[25] Leaflet - a JavaScript library for interactive maps https://leafletjs.com/ (accessed February 27, 2021).

[26] Swagger: API Documentation \& Design Tools for Teams, https://swagger.io/ (accessed February 27, 2021).

[27] TensorFlow, https://www.tensorflow.org/ (accessed February 27, 2021).

[28] Keras, https://keras.io/ (accessed February 27, 2021).

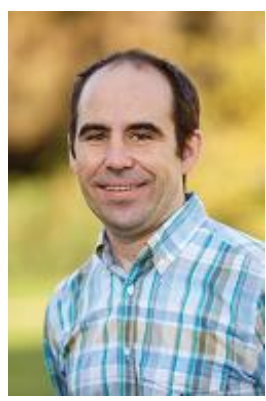

José Javier Astrain received the degree in Telecommunications Engineering (1999) and $\mathrm{PhD}$ degree in Computer Science (2004), both at the Public University of Navarre (UPNA, Spain) where he works as lecturer. He has worked in more than 70 different public and privately funded research projects, is the coinventor of four patents, coauthored more than 130 articles and contributions in international journals and conferences as well as seven book chapters. His current research interests concern wireless sensor networks, distributed systems and cloud computing. He was awarded with the Premio Talgo 2012 for Technological Innovation. He is member of the Institute of Smart Cities (UPNA).

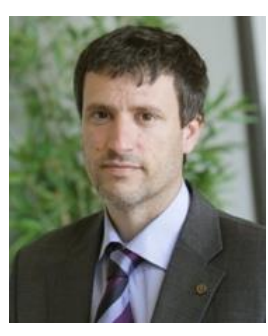

Francisco Falcone (M'05, SM'09) received the degree in telecommunication engineering and the Ph.D. degree in communication engineering from the Universidad Pública de Navarra (UPNA), Spain, in 1999 and 2005, respectively. From February 1999 to April 2000, he was a Microwave Commissioning Engineer with Siemensltaltel. From May 2000 to December 2008, he was a Radio Access Engineer with Telefónica Móviles. From January 2009 to May 2009, he was a Co-Founding Member and the Director of Tafco Metawireless, a spin-off company from UPNA. Since 2009, he os an Associate Professor with the EEC Department, UPNA. His research interests are related to computational electromagnetics applied to analysis of complex electromagnetic scenarios, with a focus on the analysis, design, and implementation of heterogeneous wireless networks to enable context aware environments.

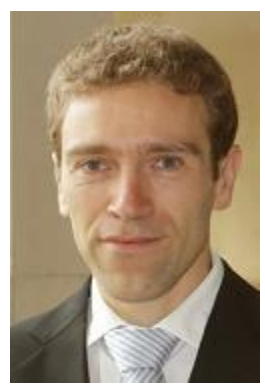

Antonio J. Lopez-Martin (M'04, SM'11) received the M.S. and Ph.D. degrees (with honors) from the Public University of Navarra, Pamplona (Spain) in 1995 and 1999, respectively. He has been a visiting professor with the New Mexico State University, Las Cruces, NM, and an invited researcher with the Swiss Federal Institute of Technology (ETH), Zurich. Currently, he is Research Director and Professor with the Public University of Navarra. His research interests include wireless transceivers and sensor interfaces with emphasis on low-voltage low-power implementations. He has published over 450 technical contributions in books, journals, and conferences. He also holds 6 international patents, leads various research projects and is consultant for local companies.

Dr. Lopez was an Associate Editor of the IEEE TRANSACTIONS ON CIRCUITS AND SYSTEMS-II: EXPRESS BRIEFS (2006-2007) and the IEEE TRANSACTIONS ON CIRCUITS AND SYSTEMS-I: REGULAR PAPERS (2008-2009), among other journals. He is a former Chair of the IEEE CASS Spain Chapter (2017-2019). He 
received the 2005 IEEE TRANSACTIONS ON EDUCATION Best Paper Award.

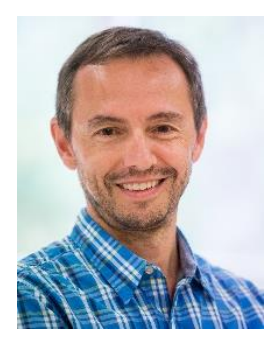

Pablo Sanchis (M'03-SM'12) received the M.Sc. degree in electrical engineering, the M.Sc. degree in management and business administration, and the Ph.D. degree in electrical engineering from the Public University of Navarre (UPNA), Pamplona, Spain, in 1994, 1995, and 2002, respectively.

From 1996 to 1998, he was a Guest Researcher with the Delft University of Technology, Delft, The Netherlands. In 1998, he joined the Department of Electrical, Electronic and Communications Engineering, Public University of Navarre, where he is now a Professor. He was the Vice Dean of the School of Industrial \& ICT Engineering and the Director of the University Unit for Research Resources and Structures. He is currently the Director of the UPNA Chair for Renewable Energies and the Head of the Research Group in Electrical Engineering, Power Electronics, and Renewable Energies. He has been involved in more than 100 research projects both with public funding and in cooperation with industry and is the coinventor of ten patents. He has supervised 10 Ph.D. theses and coauthored more than 140 articles and contributions in international journals and conferences as well as five book chapters (h-index of 32 ). His current research interests include renewable energies, power electronics, electric energy storage technologies, grid integration of renewable energies, and electric microgrids.

Dr. Sanchis received the UPNA Research Award for the Best Technical Paper in 2013 and the UPNA Excellence in Teaching Award in 2017.

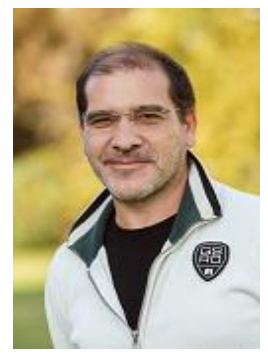

Jesús Villadangos received the Bachelor's degree in physics from the University of Basque Country, Spain, in 1991 and the Ph.D. degree in communications engineering from the Public University of Navarre, Pamplona, Spain, in 1999. In 2000, he became an Associate Professor with the Public University of Navarre. His research interests include distributed algorithms and vehicular networks. He was awarded with the Premio Talgo 2012 for Technological Innovation. He is coauthor of two Spanish patents. He is member of the Institute of Smart Cities (UPNA).

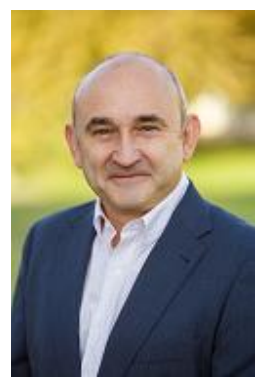

Ignacio R. Matias received the M.S. degree in Electrical and Electronic Engineering and his Ph.D. degree in Optical Fiber Sensors from the Polytechnic University of Madrid (UPM), Madrid, Spain, in 1992 and 1996, respectively. He became a Lecturer at the Public University of Navarra (Pamplona, Spain) in 1996, where presently he is a Permanent Professor. He has co-authored more than 200 journal and 250 conference papers related to sensors and passive devices and systems. He was coan IEEE Senior member. founder Editor of IEEE Sensors Journal. He is 